\title{
BEM ANALYSIS OF PLANE WAVE COUPLING TO THREE-PHASE POWER LINE
}

\author{
DRAGAN POLJAK \& DAMIR CAVKA \\ University of Split, Croatia
}

\begin{abstract}
The paper deals with BEM analysis of plane wave coupling to three-phase power line. The formulation is based on the set of Pocklington equations for arbitrarily shaped wires. The presence of a lossy ground is taken into account via rigorous Sommerfeld integral formulation. The set of Pocklington equations is solved by the Galerkin-Bubnov Indirect Boundary Element Method (GBIBEM) using isoparametric elements. Some computational examples for the currents induced along phase conductors and shield wire of the 3-phase power line are presented. The numerical results obtained via GB-IBEM are compared to the results calculated via NEC (Numerical Electromagnetic Code). The results obtained via different approaches agree satisfactorily.

Keywords: BEM analysis, antenna theory, set of Pocklington equations, power lines, current distribution.
\end{abstract}

\section{INTRODUCTION}

The knowledge of an induced current distribution along considered curved wire is a prerequisite to understand the behaviour of wire configurations such as antenna arrays, power lines, communication cables, etc. The simplest approach is to assume the waveform of the current distribution along the given wire structure [1]. Furthermore, Transmission Line (TL) approach, which neglects radiation effects, is an efficient approximation in engineering practice for a wide range of problems [1]-[5]. However, some applications still require a more accurate approach related to the solution of a corresponding integral expression arising from scattering theory (full wave models) [6].

Thus, the use of full wave model represents the most rigorous approach to analyze electromagnetic field coupling to arbitrary wire configurations in the presence of a lossy ground.

The present paper deals with the assessment of electromagnetic interference (EMI) induced along three-phase power line illuminated by the plane wave incident electric field by using the via full wave model.

The frequency domain formulation is based on the corresponding set of Pocklington integral equations for arbitrarily shape overhead wires above a lossy ground, thus providing one to account for the conductor sag. The effects of a lossy half-space are taken into account via the rigorous Sommerfeld integral approach.

Numerical solution of corresponding integro-differential equations is carried out by means of the Galerkin-Bubnov Indirect Boundary Element Method (GB-IBEM) featuring linear and quadratic isoparametric elements.

The use of integral approach and isoparametric elements for an efficient treatment of curved wires is an important advantage of the full wave model, which is not readily the case with a TL approximation.

Some illustrative numerical results pertaining to some practical scenarios involving realistic power line configurations are given in the paper. 


\section{FORMULATION VIA SET OF INTEGRO-DIFFERENTIAL EQUATIONS}

The currents $I_{n}\left(s^{\prime}\right)$ induced along the $N_{w}$ curved wires located above a lossy ground (Fig. 1) due to a plane wave electric field $E^{e x c}$ (Fig. 2) are governed by the set of Pocklington integro-differential equations [6].

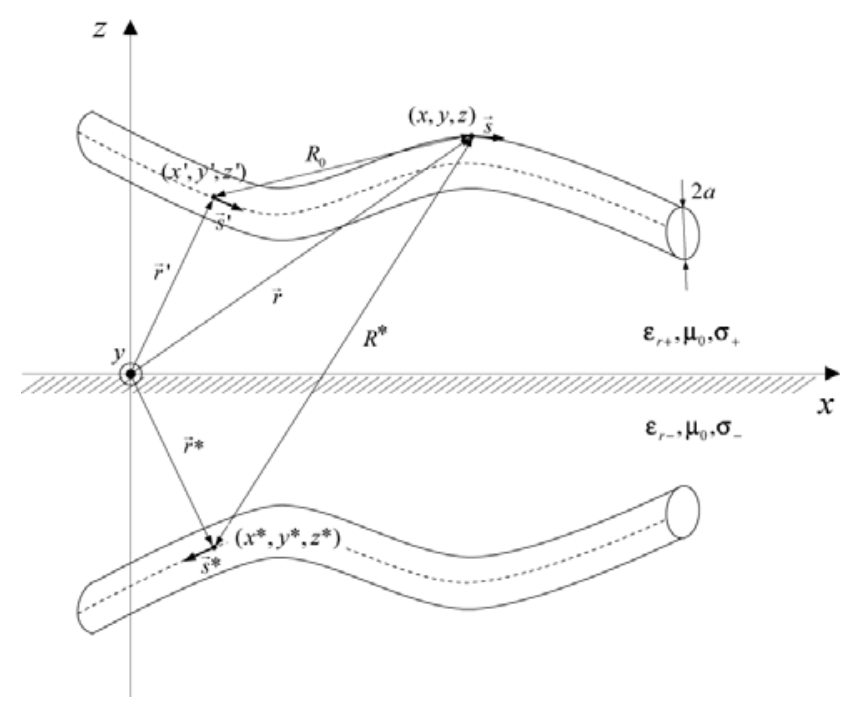

Figure 1: Buried wire of arbitrary shape and its image.

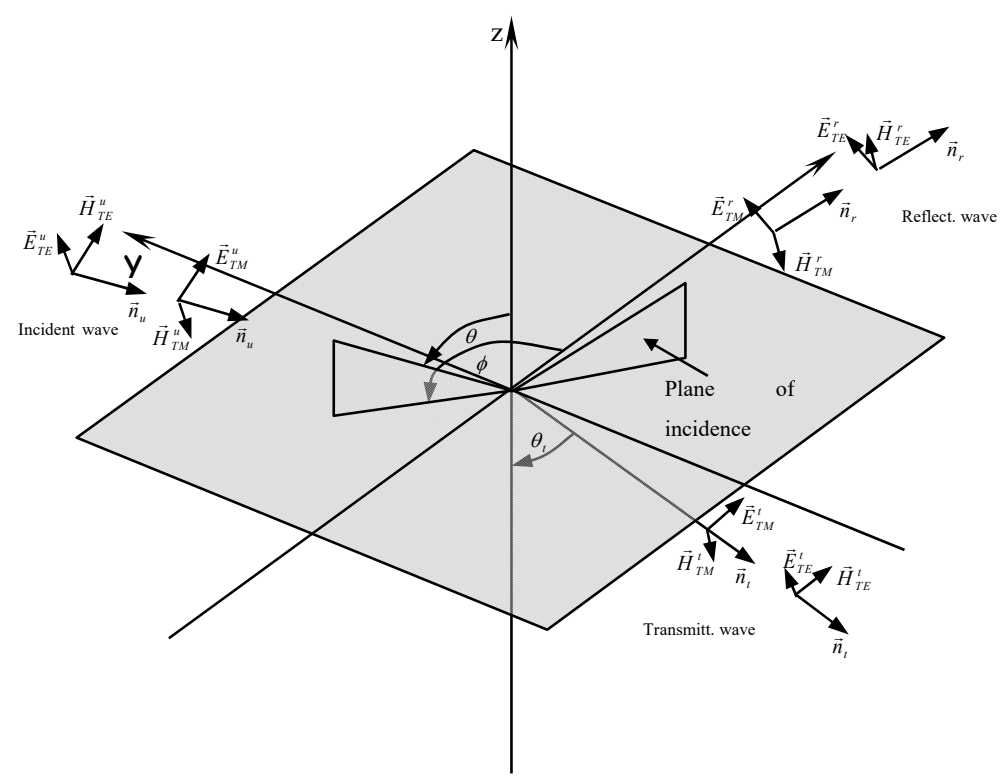

Figure 2: Incident, reflected and transmitted wave. 
The set of Pocklington equations are given by [6]

$$
E_{s m}^{e x c}(s)=-\frac{1}{j 4 \pi \omega \varepsilon_{0}} \sum_{n=1}^{N_{W}}\left[\begin{array}{l}
\int_{C_{n^{\prime}}} I_{n}\left(s^{\prime}\right) \cdot \vec{s} \cdot \vec{s}^{\prime} \cdot\left[k_{0}^{2}+\nabla \nabla\right] g_{0 n}\left(s_{m}, s_{n}{ }^{\prime}\right) d s^{\prime}+ \\
\frac{k_{g}^{2}-k_{0}^{2}}{k_{g}^{2}+k_{0}^{2}} \int_{C_{n}{ }^{\prime}} I_{n}\left(s_{n}{ }^{\prime}\right) \cdot \vec{s} \cdot \vec{s}^{*} \cdot\left[k_{0}^{2}+\nabla \nabla\right] g_{i n}\left(s_{m}, s_{n}{ }^{*}\right) d s^{\prime}+ \\
\int_{C_{n}} I_{n}\left(s^{\prime}\right) \cdot \vec{s} \cdot \vec{G}_{s}\left(s_{m}, s_{n}{ }^{\prime}\right) d s^{\prime}
\end{array}\right]+Z_{S} \cdot I_{m}(s),
$$

where the corresponding Green functions are given by:

$$
\begin{aligned}
& g_{0 m n}\left(s_{m}, s_{n}^{\prime}\right)=\frac{e^{-j k R_{1 m n}}}{R_{1 m n}}, \\
& g_{\text {imn }}\left(s_{m}, s_{n}^{\prime}\right)=\frac{e^{-j k R_{2 m n}}}{R_{2 m n}},
\end{aligned}
$$

where $R_{1 \mathrm{mn}}$ and $R_{2 \mathrm{mn}}$ are distances from the source point (from real and image wire axis, respectively) to the observation point (at the real wire surface)

$$
\vec{G}_{s}\left(s, s^{\prime}\right)=\left(\vec{e}_{x} \cdot \vec{s}^{\prime}\right) \cdot\left(G_{\rho}^{H} \cdot \vec{e}_{\rho}+G_{\phi}^{H} \cdot \vec{e}_{\phi}+G_{z}^{H} \cdot \vec{e}_{z}\right)+\left(\vec{e}_{z} \cdot \vec{s}^{\prime}\right) \cdot\left(G_{\rho}^{V} \cdot \vec{e}_{\rho}+G_{z}^{V} \cdot \vec{e}_{z}\right)
$$

while the related wave numbers are:

$$
\begin{gathered}
k_{0}^{2}=\omega^{2} \mu_{0} \varepsilon_{0}, \\
k_{g}^{2}=\omega^{2} \mu_{0} \varepsilon_{e f f}=\omega^{2} \mu_{0}\left(\varepsilon_{0} \varepsilon_{r g}-j \frac{\sigma_{g}}{\omega}\right),
\end{gathered}
$$

where $\varepsilon_{\mathrm{rg}}$ and $\sigma_{\mathrm{g}}$ are relative permittivity and conductivity of the ground, respectively, and $\omega$ is the frequency of interest. The kernel terms are, as follows:

$$
\begin{gathered}
G_{\rho}^{V}=\frac{\partial^{2}}{\partial \rho \partial z} k_{g}^{2} V^{R} \\
G_{z}^{V}=\left(\frac{\partial^{2}}{\partial z^{2}}+k_{0}^{2}\right) k_{g}^{2} V^{R}, \\
G_{\rho}^{H}=\cos \phi\left(\frac{\partial^{2}}{\partial \rho^{2}} k_{0}^{2} V^{R}+k_{0}^{2} U^{R}\right), \\
G_{\phi}^{H}=-\sin \phi\left(\frac{1}{\rho} \frac{\partial}{\partial \rho} k_{0}^{2} V^{R}+k_{0}^{2} U^{R}\right), \\
G_{z}^{H}=-j 4 \pi \omega \varepsilon_{0} \cos \phi G_{\rho}^{V} .
\end{gathered}
$$

And the Sommerfeld integral terms are given by: 


$$
\begin{gathered}
U^{R}=\int_{0}^{\infty} D_{1}(\lambda) e^{-\gamma_{0}\left|z+z^{\prime}\right|} J_{0}(\lambda \rho) \lambda d \lambda, \\
V^{R}=\int_{0}^{\infty} D_{2}(\lambda) e^{-\gamma_{0}\left|z+z^{\prime}\right|} J_{0}(\lambda \rho) \lambda d \lambda, \\
D_{1}(\lambda)=\frac{2}{\gamma_{0}+\gamma_{g}}-\frac{2 k_{0}^{2}}{\gamma_{0}\left(k_{0}^{2}+k_{g}^{2}\right)}, \\
D_{2}(\lambda)=\frac{2}{k_{g}^{2} \gamma_{0}+k_{0}^{2} \gamma_{g}}-\frac{2}{\gamma_{0}\left(k_{0}^{2}+k_{g}^{2}\right)}, \\
\gamma_{0}=\sqrt{\lambda^{2}-k_{0}^{2}} ; \quad \gamma_{g}=\sqrt{\lambda^{2}-k_{g}^{2}},
\end{gathered}
$$

where $J_{0}$ denotes the Bessel function. The set of Pocklington IEs is handled via the GBIBEM with isoparametric elements.

\section{NUMERICAL SOLUTION}

The set of Pocklington IEs is handled via the GB-IBEM with isoparametric elements. The unknown current along the $n$-th wire segment is expressed by a sum of independent basis functions with unknown complex coefficients:

$$
I_{n}^{e}\left(s^{\prime}\right)=\sum_{i=1}^{n} I_{n i} f_{n i}\left(s^{\prime}\right)=\{f\}_{n}^{T}\{I\}_{n} .
$$

The use of isoparametric elements yields:

$$
I_{n}^{e}(\zeta)=\sum_{i=1}^{n} I_{n i} f_{n i}(\zeta)=\{f\}_{n}^{T}\{I\}_{n}
$$

Having performed BEM discretization procedure the set of coupled Pocklington equations is transformed into the following matrix equation

$$
\sum_{n=1}^{N_{w}} \sum_{\mathrm{i}=1}^{N_{n}}[Z]_{\mathrm{ji}}^{\mathrm{e}}\{I\}_{\mathrm{i}}^{\mathrm{e}}=\{\mathrm{V}\}_{\mathrm{j}}^{\mathrm{e}}, \mathrm{m}=1,2, \ldots, N_{w} ; \quad \mathrm{j}=1,2, \ldots, N_{m},
$$

where the local matrix and right-side vector are given by:

$$
\begin{aligned}
& {[Z]_{i j}^{e}=-\int_{-1}^{1} \int_{-1}^{1}\{D\}_{j}\left\{D^{\prime}\right\}_{i}^{T} g_{0 n m}\left(s_{n}, s_{m}^{\prime}\right) \frac{d s_{m}^{\prime}}{d \xi^{\prime}} d \xi^{\prime} \frac{d s_{n}}{d \xi} d \xi} \\
& +k_{0}^{2} \vec{s}_{n} \cdot \vec{s}_{m}^{\prime} \int_{-1}^{1} \int_{-1}^{1}\{f\}_{j}\left\{f^{\prime}\right\}_{i}^{T} g_{0 n m}\left(s_{n}, s_{m}^{\prime}\right) \frac{d s_{m}^{\prime}}{d \xi^{\prime}} d \xi^{\prime} \frac{d s_{n}}{d \xi} d \xi- \\
& -\frac{k_{g}^{2}-k_{0}^{2}}{k_{g}^{2}+k_{0}^{2}} \int_{-1-1}^{1} \int_{-1}^{1}\{D\}_{j}\left\{D^{\prime}\right\}_{i}^{T} g_{i n m}\left(s_{n}, s_{m}^{*}\right) \frac{d s_{m}^{\prime}}{d \xi^{\prime}} d \xi^{\prime} \frac{d s_{n}}{d \xi} d \xi \\
& +\frac{k_{g}^{2}-k_{0}^{2}}{k_{g}^{2}+k_{0}^{2}} k_{0}^{2} \vec{s}_{n} \cdot \vec{s}_{m} \int_{-1-1}^{1} \int_{-1}^{1}\{f\}_{j}\left\{f^{\prime}\right\}_{i}^{T} g_{i n m}\left(s_{n}, s_{m}^{*}\right) \frac{d s_{m}^{\prime}}{d \xi^{\prime}} d \xi^{\prime} \frac{d s_{n}}{d \xi} d \xi+ \\
& +\vec{s}_{m}^{1} \int_{-1-1}^{1} \int_{-1}^{1}\{f\}_{j}\left\{f^{\prime}\right\}_{i}^{T} \vec{G}_{s n m}\left(s_{n}, s_{m}^{*}\right) \frac{d s_{m}^{\prime}}{d \xi^{\prime}} d \xi^{\prime} \frac{d s_{n}}{d \xi} d \xi+\frac{j}{4 \pi \omega \varepsilon_{0}} \int_{-1}^{1} Z_{T}^{\prime}\{f\}_{j}\left\{f^{\prime}\right\}_{j}^{T} \frac{d s_{n}}{d \xi},
\end{aligned}
$$




$$
\{V\}_{j}^{n}=-j 4 \pi \omega \varepsilon_{0} \int_{-1}^{1} E_{s_{n}}^{e x c}\left(s_{n}\right)\{f\}_{j} \frac{d s_{n}}{d \xi} d \xi_{n} .
$$

Note that the physical meaning of (20) is the mutual impedance, while (21) represents the voltage.

\section{COMPUTATIONAL EXAMPLE}

The computational example is related to a plane wave $(\mathrm{f}=5 \mathrm{MHz})$ coupling to 3 -phase power line, shown in Fig. 3, consisting of 4 wires (phase conductors+shield wire) above a lossy ground $\left(\varepsilon_{r}=10, \sigma=0.001 \mathrm{~S} / \mathrm{m}\right)$. The separation between towers iz $300 \mathrm{~m}$. The radius of perfectly conducting (PEC) conductors is $a=5 \mathrm{~mm}$.

More details about this particular power line configuration can be found elsewhere, e.g. in [7].

\subsection{Case No 1: Straight conductors}

The power line system consisting of straight conductors illuminated by the plane wave incidence $\left(\alpha=0^{\circ}, \theta=60^{\circ}\right.$ and $\left.\varphi=30^{\circ}\right)$ with amplitude $E_{0}=1 \mathrm{~V} / \mathrm{m}$, and frequency $f=5 \mathrm{MHz}$ is considered. Fig. 4(a)-(d) show the real and imaginary parts of the current distribution along the wires calculated via GB-IBEM and compared to the results computed via NEC software package [8].

An excellent agreement between the results obtained via GB-IBEM and NEC is considered.

\subsection{Case No 2: Curved conductors}

In present example the wire sag ( $23 \mathrm{~m}$ for phase conductors, $13.5 \mathrm{~m}$ for shield wire) is taken into account. The power line system is illuminated by the plane wave incidence $\left(\alpha=0^{\circ}\right.$, $\theta=0^{\circ}$ and $\varphi=0$ ) with amplitude $E_{0}=1 \mathrm{~V} / \mathrm{m}$, and frequency $f=5 \mathrm{MHz}$. Fig. 5 shows the curresnt distribution along the wires calculated via GB-IBEM (linear and quadratic elements) and compared to the results obtained via NEC software package.

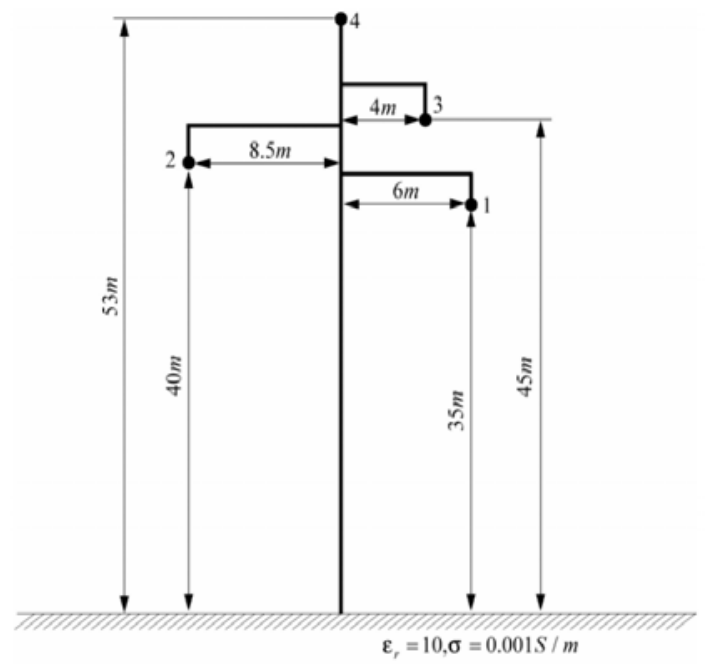

Figure 3: Three-phase power line system. 

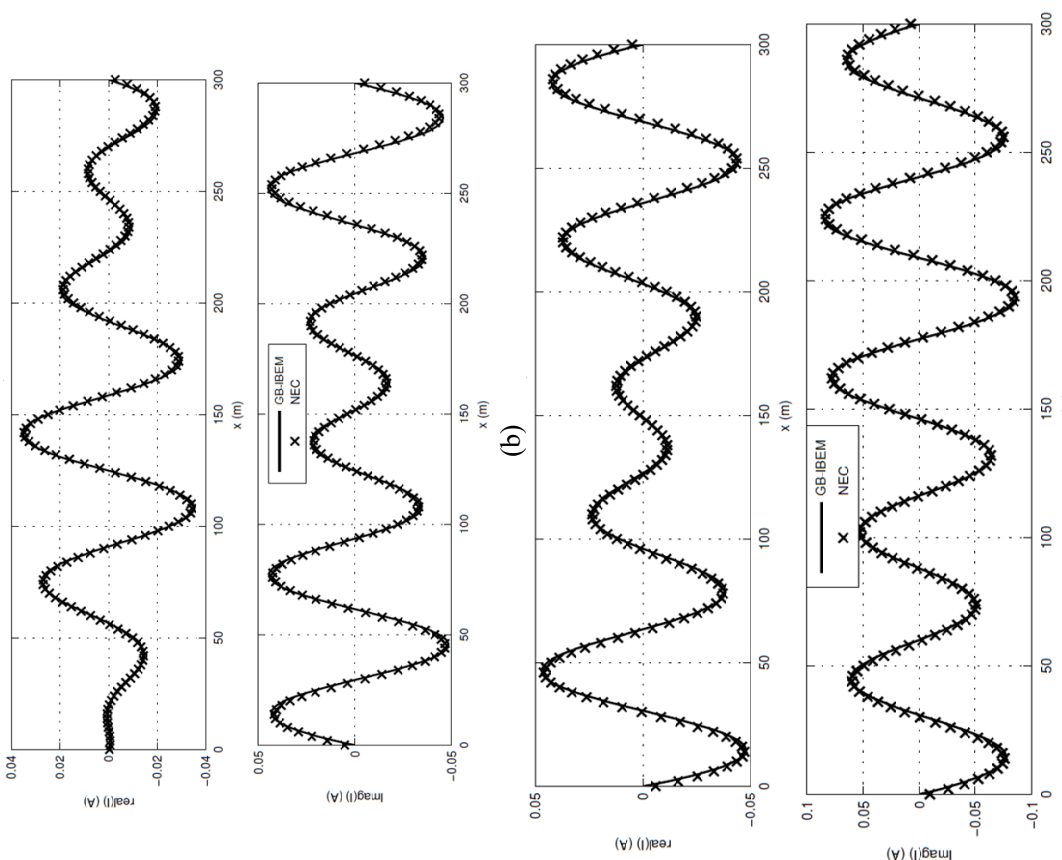

. 동
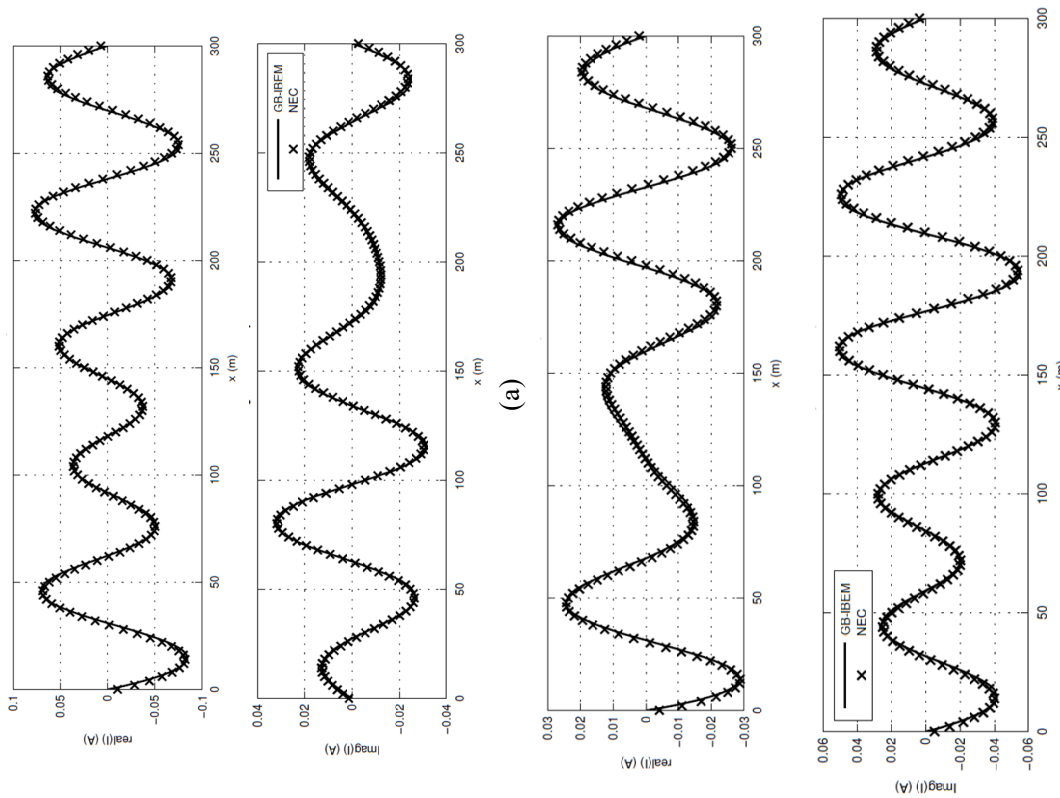

은

을 흘

일

蛋

긍

를 ․․․

需

$\mathscr{2}$

Ð0)

낭 수을

늘음

oi 홍

.

귱

ส

ब

(ีํํำ

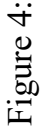



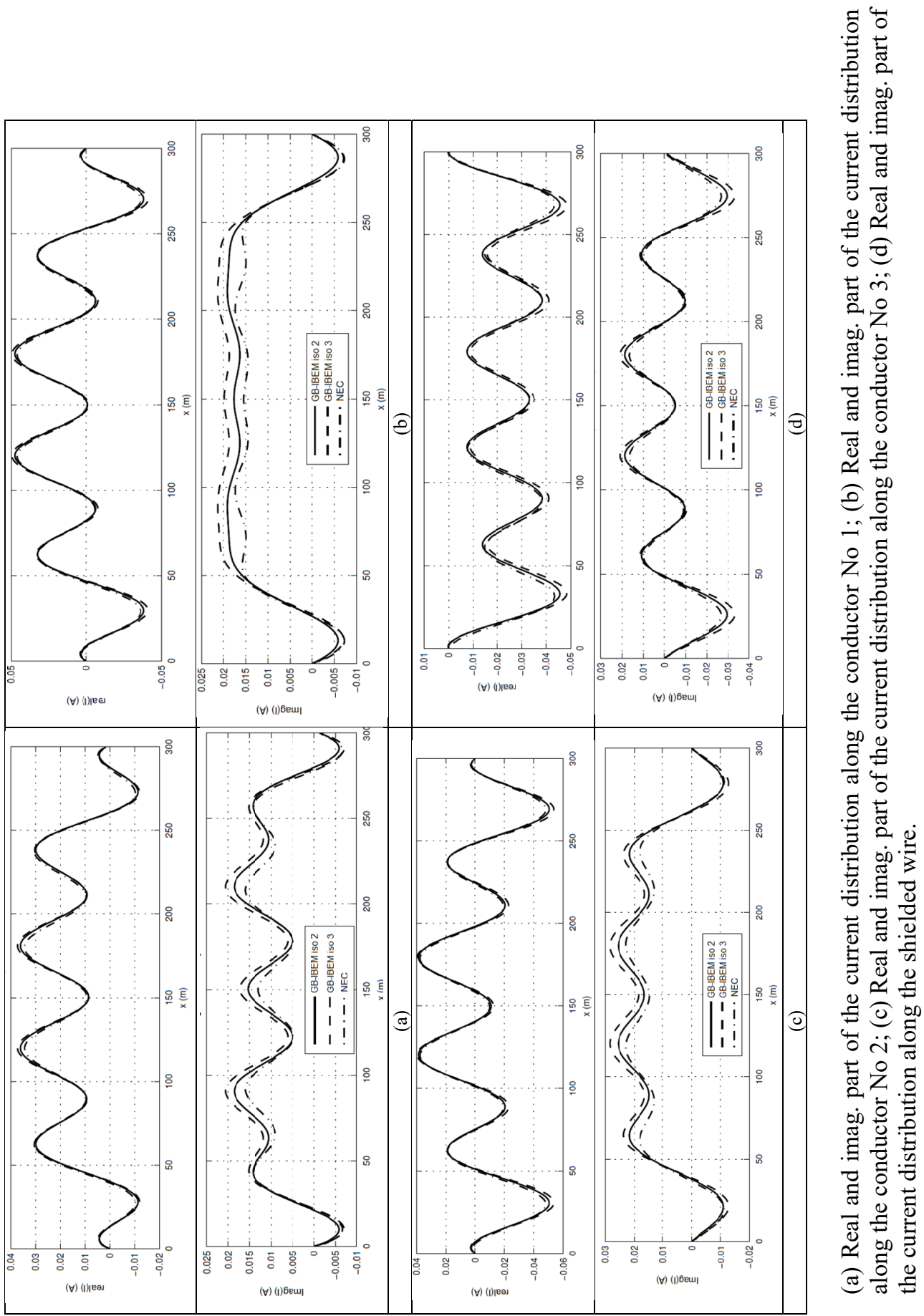

$\ddot{\ddot{n}}$ 


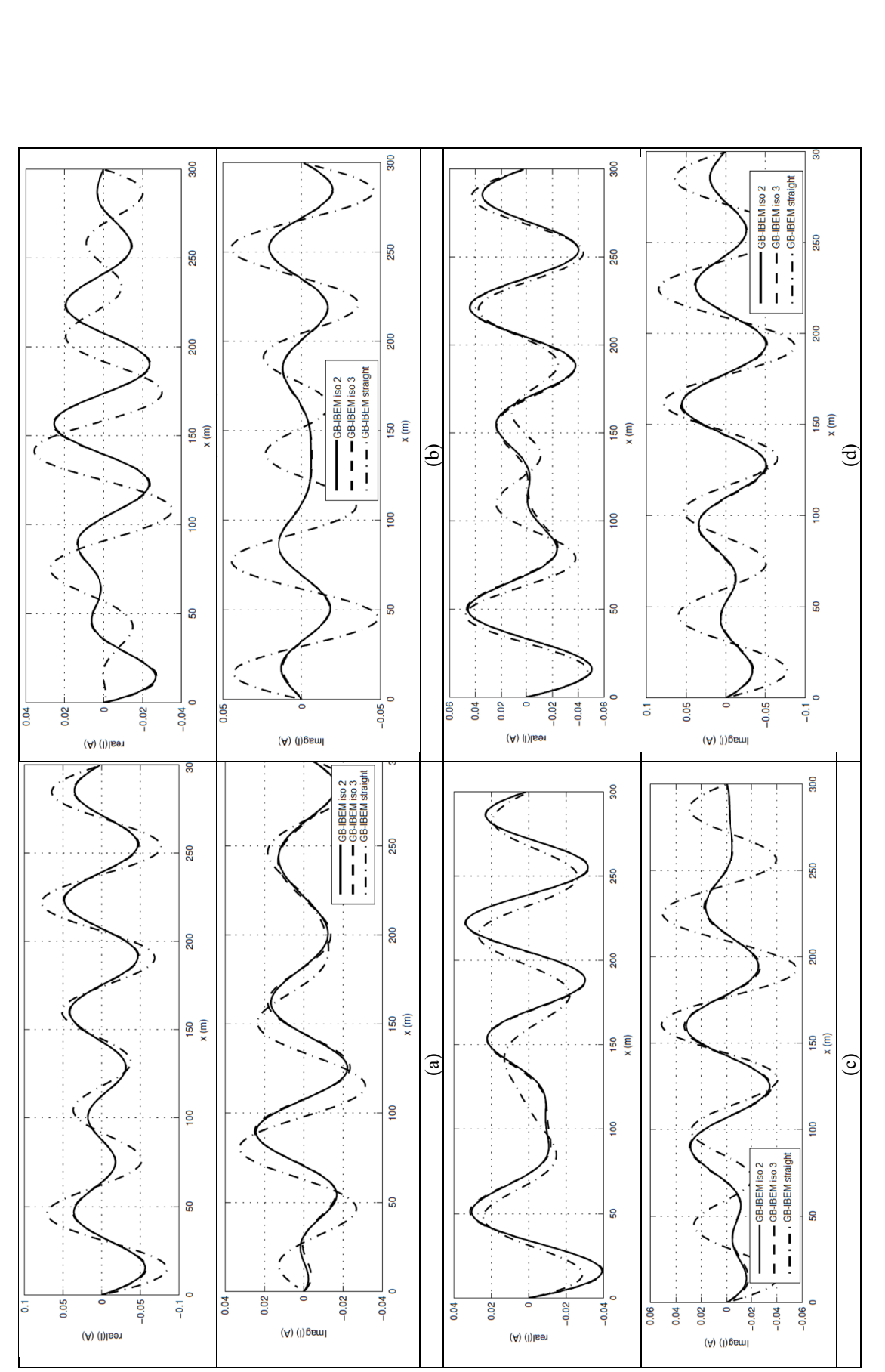

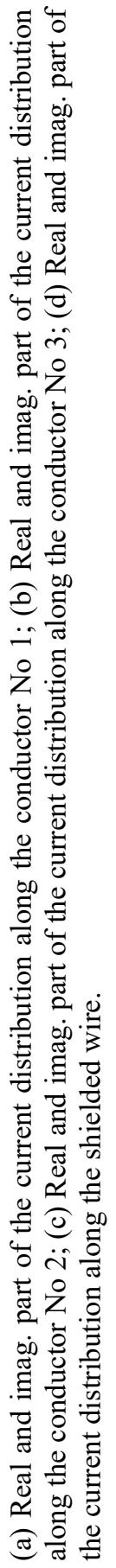

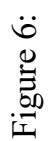


Some discrepancies are noticeable in imaginary part of current distribution along the conductors.

\subsection{Case No 3: straight and curved conductors}

Fig. 6 shows the comparison of currents induced on the power line system for the case of straight and curved conductors respectively. The excitation is unit amplitdue plane wave $\left(\alpha=0^{\circ}, \theta=60^{\circ}\right.$ and $\left.\varphi=30^{\circ}\right)$ of frequency $5 \mathrm{MHz}$. The current distribution along the straight conductors is calculated by using linear interpolation only, while the current distribution along the curved wires is calculated by using linear and quadratic approximation, respectively.

The influence of the conductor sag is clearly demonstrated in Fig. 6(a)-(d).

\section{CONCLUDING REMARKS}

BEM modeling of electromagnetic field coupling to 3-phase power line is carried out in this work. The formulation is based on the set of Pocklington integro-differential equations for curved wires. The presence of a lossy half-space is taken into account via rigorous Sommerfeld integrals.

The set of Pocklington integro-differential equations is handled via the frequency domain variant of the GB-IBEM featuring the use of isoparametric elements.

Some illustrative numerical results for the currents induced along phase conductors and shield wire of the 3-phase power line are given in the paper.

The numerical results obtained via GB-IBEM are compared to the results calculated via commercial software package NEC (Numerical Electromagnetic Code) for the case of straight conductors. The results obtained via different approaches agree satisfactorily. The influence of the conductor sag becomes clearly visible when the results obtained for straight and curved conductors are compared.

\section{REFERENCES}

[1] Tesche, F.M., Ianoz, M. \& Karlsson, T., EMC Analysis Methods and Computational Models, Wiley Interscience: New York, 1997.

[2] Agrawal, A.K., Price, H.J. \& Gurbaxani, S.H., Transient response of multiconductor transmission lines excited by a nonuniform electromagnetic field. IEEE Transactions on Electromagnetic Compatibility, 22(2), pp. 119-129, 1980.

DOI: $10.1109 /$ temc.1980.303824.

[3] Poljak, D., Advanced Modeling in Computational Electromagnetic Compatibility, Wiley: New York, 2007.

[4] Poljak, D. \& Khamlichi Drissi, K.El., Electromagnetic field coupling to overhead wire configurations: antenna model versus transmission line approach. International Journal of Antennas and Propagation, 2012, pp. 1-18, 2012.

DOI: $10.1155 / 2012 / 730145$.

[5] Bridges G.E. \& Shafai, L., Plane wave coupling to multiple conductor transmission lines above a lossy earth. IEEE Transactions on Electromagnetic Compatibility, 31(1), pp. 21-33, 1989. DOI: 10.1109/15.19904.

[6] Poljak, D. \& Cavka, D., Electromagnetic compatibility aspects of wind turbine analysis and design. Properties and Characterization of Modern Materials, eds. A. Ochsner \& H. Altenbach, Springer, 2017. 
72 Boundary Elements and other Mesh Reduction Methods XLI

[7] Kraljevic, S., Poljak, D. \& Doric, V., A simplified method for the assessment of ELF magnetic fields from three-phase power lines, Proc. Boundary Elements XXVII, Orlando, USA, pp. 551-562, 2005.

[8] Burke, G.J., Poggio, A.J., Logan, I.C. \& Rockway, J.W., Numerical electromagnetics code: A program for antenna system analysis. Proc. EMC 1979 Symp., Rotterdam, The Netherlands, pp. 89-94. 